\title{
Phase Equilibria of Confined Fluids in Nanopores of Tight and Shale Rocks Considering the Effect of Capillary Pressure and Adsorption Film
}

\author{
Xiaohu Dong, ${ }^{*}{ }^{1,2}$ Huiqing Liu, ${ }^{1}$ Jirui Hou, ${ }^{1}$ Keliu Wu, ${ }^{2}$ Zhangxin Chen ${ }^{1,2}$ \\ 1 China University of Petroleum, Beijing, 102249, China \\ 2 Department of Chemical and Petroleum Engineering, University of Calgary, Calgary, T2N 1N4 Alberta, \\ Canada \\ *Corresponding Author, Tel: +1.403.708.6582; Email: donghu820@163.com
}


Table S1 The parameters of Langmuir model for methane, ethane, n-pentane, and n-butane.

\begin{tabular}{|c|c|c|c|c|c|c|}
\hline No. & Adsorbate & Adsorbent & $\begin{array}{c}\text { Temperature } \\
\text { (K) }\end{array}$ & $\begin{array}{c}\mathbf{V}_{m} \\
(\mathrm{mmol} / \mathrm{g})\end{array}$ & $\begin{array}{c}\mathrm{b} \\
\left(\mathrm{MPa}^{-1}\right)\end{array}$ & References \\
\hline 1 & $\mathrm{CH}_{4}$ & $\mathrm{ACF}$ & 293 & 5.823 & 1.734 & Choi et al. 2003 \\
\hline 2 & $\mathrm{CH}_{4}$ & $\mathrm{ACF}$ & 303 & 5.565 & 1.453 & Choi et al. 2003 \\
\hline 3 & $\mathrm{CH}_{4}$ & $\mathrm{ACF}$ & 313 & 5.520 & 1.204 & Choi et al. 2003 \\
\hline 4 & $\mathrm{CH}_{4}$ & $\mathrm{ACF}$ & 333 & 4.759 & 0.9176 & Gusev et al. 1996. \\
\hline 5 & $\mathrm{CH}_{4}$ & $\mathrm{ACF}$ & 373 & 3.967 & 0.6560 & Gusev et al. 1996. \\
\hline 6 & $\mathrm{CH}_{4}$ & MCM-41 silica & 303 & 5.860 & 0.2007 & Yun et al 2002 \\
\hline 7 & $\mathrm{CH}_{4}$ & MCM-41 silica & 373 & 5.159 & 0.09060 & Yun et al 2002 \\
\hline 8 & $\mathrm{CH}_{4}$ & Silicalite & 273 & 15.18 & 1.182 & Song et al. 2007 \\
\hline 9 & $\mathrm{CH}_{4}$ & Silicalite & 298 & 8.802 & 0.7562 & Song et al. 2007 \\
\hline 10 & $\mathrm{C}_{2} \mathrm{H}_{6}$ & $\mathrm{ACF}$ & 293 & 6.51 & 13.30 & Choi et al. 2003 \\
\hline 11 & $\mathrm{C}_{2} \mathrm{H}_{6}$ & $\mathrm{ACF}$ & 303 & 6.294 & 9.807 & Choi et al. 2003 \\
\hline 12 & $\mathrm{C}_{2} \mathrm{H}_{6}$ & $\mathrm{ACF}$ & 313 & 6.066 & 8.648 & Choi et al. 2003 \\
\hline 13 & $\mathrm{C}_{2} \mathrm{H}_{6}$ & $\mathrm{ACF}$ & 373 & 4.661 & 3.183 & Gusev et al. 1996 \\
\hline 14 & $\mathrm{C}_{2} \mathrm{H}_{6}$ & MCM-41 silica & 303 & 11.15 & 0.4490 & Yun et al. 2002 \\
\hline 15 & $\mathrm{C}_{2} \mathrm{H}_{6}$ & MCM-41 silica & 373 & 3.685 & 0.4910 & Yun et al. 2002 \\
\hline 16 & $\mathrm{n}-\mathrm{C}_{3} \mathrm{H}_{8}$ & Silicalite & 348 & 0.9305 & 203.3 & Song et al. 2007 \\
\hline 17 & $\mathrm{n}-\mathrm{C}_{3} \mathrm{H}_{8}$ & Silicalite & 373 & 0.8508 & 127.0 & Song et al. 2007 \\
\hline 18 & $\mathrm{n}-\mathrm{C}_{3} \mathrm{H}_{8}$ & Silicalite & 398 & 0.8228 & 60.23 & Song et al. 2007 \\
\hline 19 & $\mathrm{n}-\mathrm{C}_{3} \mathrm{H}_{8}$ & Silicalite & 423 & 0.7881 & 32.12 & Song et al. 2007 \\
\hline 20 & $n-C_{4} H_{10}$ & Silicalite & 323 & 1.469 & 4166 & Song et al. 2007 \\
\hline 21 & $\mathrm{n}-\mathrm{C}_{4} \mathrm{H}_{10}$ & Silicalite & 348 & 1.329 & 1198 & Song et al. 2007 \\
\hline 22 & $n-C_{4} H_{10}$ & Silicalite & 373 & 1.150 & 459.7 & Song et al. 2007 \\
\hline 23 & $n-C_{4} H_{10}$ & Silicalite & 398 & 0.9688 & 227.6 & Song et al. 2007 \\
\hline
\end{tabular}


Table S2 The parameters of BET model for the adsorbates of n-pentane, n-hexane and n-octane.

\begin{tabular}{|cccccccc|}
\hline No. & Adsorbate & Adsorbent & $\begin{array}{c}\text { Temperature } \\
(\mathbf{K})\end{array}$ & $\begin{array}{c}\boldsymbol{P}_{\boldsymbol{o}} \\
\text { (MPa) }\end{array}$ & $\begin{array}{c}\mathbf{V}_{\boldsymbol{m}} \\
(\mathbf{m m o l} / \mathbf{g})\end{array}$ & $\boldsymbol{C}$ & References \\
\hline 1 & $\mathrm{n}-\mathrm{C}_{5} \mathrm{H}_{12}$ & Silicalite & 323 & 0.1595 & 59.14 & 2113 & Song et al. 2007 \\
2 & $\mathrm{n}-\mathrm{C}_{5} \mathrm{H}_{12}$ & Silicalite & 348 & 0.3204 & 13.08 & 1911 & Song et al. 2007 \\
3 & $\mathrm{n}-\mathrm{C}_{5} \mathrm{H}_{12}$ & Silicalite & 373 & 0.5810 & 2.957 & 1127 & Song et al. 2007 \\
4 & $\mathrm{n}-\mathrm{C}_{5} \mathrm{H}_{12}$ & Silicalite & 423 & 1.558 & 0.2969 & 765.5 & Song et al. 2007 \\
5 & $\mathrm{n}-\mathrm{C}_{5} \mathrm{H}_{12}$ & Silicalite & 450 & 2.457 & 0.1940 & 265.7 & Song et al. 2007 \\
6 & $\mathrm{n}-\mathrm{C}_{5} \mathrm{H}_{12}$ & Carbonaceous & 333 & 0.2114 & 3.156 & 1102 & Do and Do, 2002 \\
7 & $\mathrm{n}-\mathrm{C}_{5} \mathrm{H}_{12}$ & Carbonaceous & 353 & 0.3617 & 3.041 & 1096 & Do and Do, 2002 \\
8 & $\mathrm{n}-\mathrm{C}_{5} \mathrm{H}_{12}$ & Carbonaceous & 423 & 1.558 & 2.619 & 477.3 & Do and Do, 2002 \\
9 & $\mathrm{n}-\mathrm{C}_{6} \mathrm{H}_{14}$ & Silicalite & 336 & 0.07637 & 1.382 & 1808 & Song and Rees 1997 \\
10 & $\mathrm{n}-\mathrm{C}_{6} \mathrm{H}_{14}$ & Silicalite & 353 & 0.1424 & 1.132 & 2945 & Song and Rees 1997 \\
11 & $\mathrm{n}-\mathrm{C}_{6} \mathrm{H}_{14}$ & Silicalite & 373 & 0.2413 & 0.8970 & 2787 & Song and Rees 1997 \\
12 & $\mathrm{n}-\mathrm{C}_{6} \mathrm{H}_{14}$ & Silicalite & 398 & 0.4394 & 0.8019 & 2078 & Song and Rees 1997 \\
13 & $\mathrm{n}-\mathrm{C}_{6} \mathrm{H}_{14}$ & Silicalite & 423 & 0.7352 & 0.7205 & 1542 & Song and Rees 1997 \\
14 & $\mathrm{n}-\mathrm{C}_{6} \mathrm{H}_{14}$ & Silicalite & 448 & 1.066 & 0.6914 & 657.4 & Song and Rees 1997 \\
15 & $\mathrm{n}-\mathrm{C}_{8} \mathrm{H}_{18}$ & ACF & 303 & 0.002461 & 3.804 & 876.3 & Matsumoto et al. 1997 \\
\hline
\end{tabular}

Table S3 Physical parameters and binary interaction coefficients of hydrocarbon mixture.

\begin{tabular}{|c|c|c|c|c|c|c|c|c|c|c|}
\hline \multirow{2}{*}{ Component } & \multirow{2}{*}{$\begin{array}{c}\text { Critical } \\
\text { pressure, } \\
\text { MPa }\end{array}$} & \multirow{2}{*}{$\begin{array}{c}\text { Critical } \\
\text { temperature, } \\
\text { K }\end{array}$} & \multirow{2}{*}{$\begin{array}{c}\text { Acentric } \\
\text { Factor }\end{array}$} & \multirow{2}{*}{$\begin{array}{l}\text { Molar } \\
\text { weight }\end{array}$} & \multicolumn{6}{|c|}{ Binary interaction coefficient } \\
\hline & & & & & $\mathrm{CH}_{4}$ & $\mathrm{C}_{2} \mathrm{H}_{6}$ & $\mathbf{C}_{3} \mathbf{H}_{8}$ & $n-C_{5} H_{12}$ & $\mathrm{n}-\mathrm{C}_{7} \mathrm{H}_{16}$ & $n-C_{10} H_{22}$ \\
\hline $\mathrm{CH}_{4}$ & 4.60 & 190.6 & 0.0102 & 16.04 & 0 & 0.005 & 0.010 & 0.030 & 0.035 & 0.045 \\
\hline $\mathrm{C}_{2} \mathrm{H}_{6}$ & 4.88 & 305.4 & 0.1028 & 30.07 & 0.005 & 0 & 0.005 & 0.010 & 0.020 & 0.020 \\
\hline $\mathbf{C}_{3} \mathbf{H}_{8}$ & 4.25 & 369.8 & 0.1520 & 44.09 & 0.010 & 0.005 & 0 & 0.020 & 0.005 & 0.005 \\
\hline$n-C_{5} H_{12}$ & 3.37 & 469.6 & 0.2510 & 72.15 & 0.030 & 0.010 & 0.020 & 0 & 0 & 0 \\
\hline $\mathrm{n}-\mathrm{C}_{7} \mathrm{H}_{16}$ & 2.74 & 540.2 & 0.3510 & 100.20 & 0.035 & 0.020 & 0.005 & 0 & 0 & 0 \\
\hline$n-C_{10} H_{22}$ & 2.11 & 617.6 & 0.490 & 142.29 & 0.045 & 0.020 & 0.005 & 0 & 0 & 0 \\
\hline
\end{tabular}




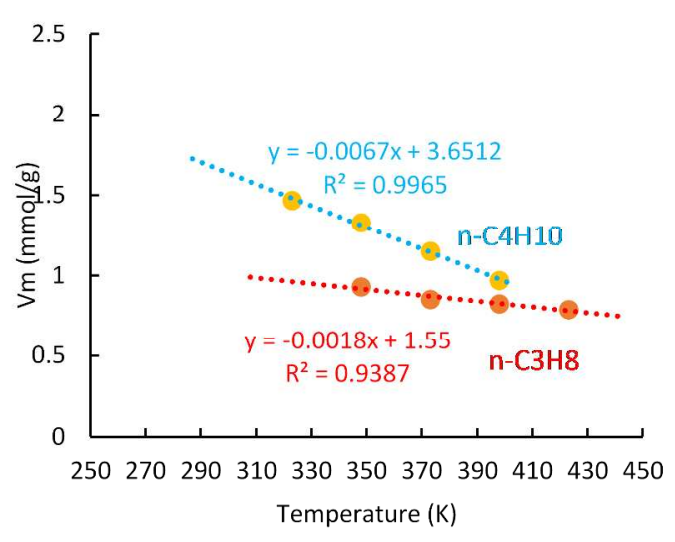

(a) $V_{m}$ varies with temperature.

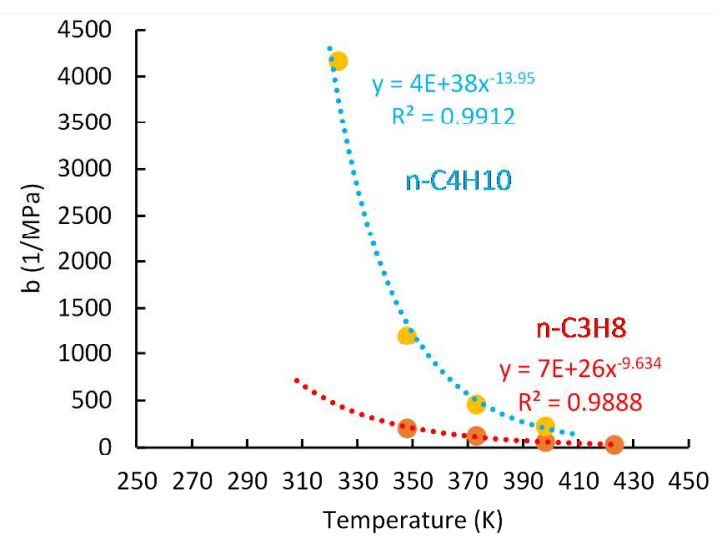

(b) b varies with temperature.

\section{Figure S1. The Langmuir adsorption parameters versus temperature.}

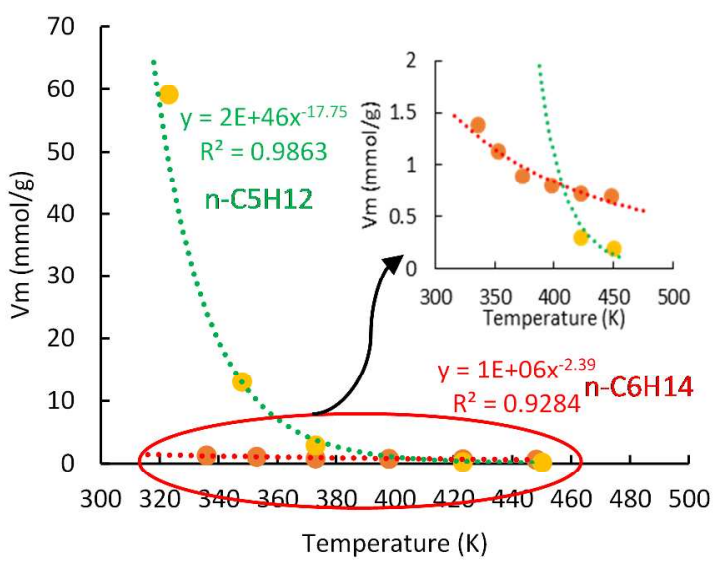

(a) $V_{m}$ varies with temperature.

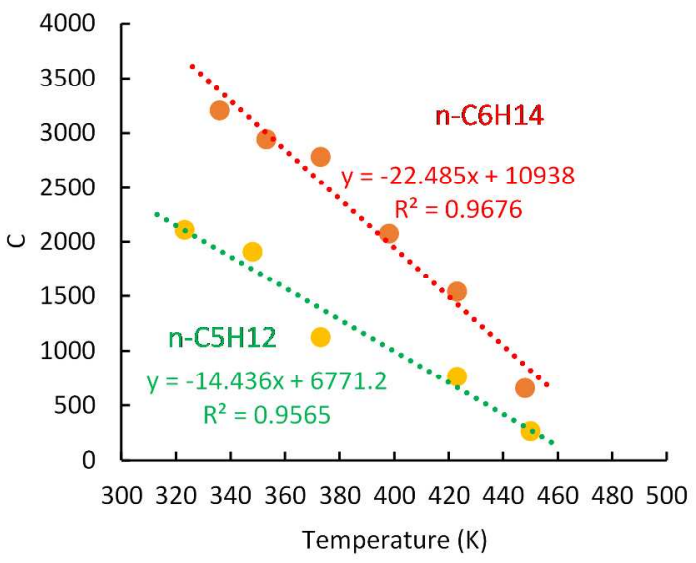

(b) $C$ varies with temperature.

Figure S2. The BET adsorption parameters versus temperature. 